\title{
InGaAsP photonic band gap crystal membrane microresonators*
}

\author{
A. Scherer, ${ }^{\text {a) }}$ O. Painter, B. D'Urso, R. Lee, and A. Yariv \\ Caltech, Laboratory of Applied Physics, Pasadena, California 91125
}

(Received 29 May 1998; accepted 16 September 1998)

\begin{abstract}
We have microfabricated two-dimensional (2D) photonic band gap structures in a thin slab of dielectric material to define reflectors and high- $Q$ microresonators. By selectively omitting holes from the 2D photonic crystal, optical microcavities, and in-plane microresonator switches can be defined. We have designed this structure with a finite difference time domain approach, and demonstrate the effect of lithographic 2D band gap tuning on the emission spectra of InGaAs/ InGaAsP multiple quantum well material emission wavelength of $1.55 \mu \mathrm{m}$. (C) 1998 American Vacuum Society. [S0734-211X(98)12106-6]
\end{abstract}

\section{INTRODUCTION}

Fabrication techniques for constructing two-dimensional (2D) photonic band gap crystals make it possible to control the propagation of light within a semiconductor material and to create optical microcavities with very small volumes. Confinement of light in the vertical direction can be obtained by using a suspended membrane of high refractive index material surrounded by air on both sides. A 2D photonic crystal in the horizontal plane can then be defined into this waveguiding slab to form an optical microcavity, and waveguides in the photonic crystals can be used to connect adjacent optical devices. ${ }^{1}$ Microcavities based on photonic crystals can therefore be used as light emitting devices with very small mode volumes, efficient microlaser sources, and in-plane microresonator networks. ${ }^{2,3}$

Here we design and fabricate such in-plane membrane microresonators with the desired freedom in the geometrical design of the cavities as well as the possibility of efficient coupling. We consider the use of a triangular array of air holes in a slab of material with a high dielectric constant, which has been shown to exhibit a band gap for both transverse electric (TE) and transverse magnetic (TM) polarizations. ${ }^{1}$ The InGaAs/InGaAsP material system was chosen since it does not suffer from large surface recombination losses and is relatively easy to microfabricate structures into. ${ }^{3}$ It has previously ${ }^{3,4}$ been used to demonstrate optically and electrically pumped whispering gallery mode microdisk lasers with diameters down to $2 \mu \mathrm{m}$ and cavity thickness comparable to our designs.

\section{CAVITY DESIGN AND FABRICATION}

\section{A. Optical microcavity design}

The cavity structures are formed by combining localization in a thin high refractive index slab due to total internal reflection, and lateral confinement with the use of a $2 \mathrm{D}$ photonic crystal. The high index slab in our case is designed to be half a wavelength thick in order to shrink the optical mode volume as much as possible. 2D band structure

\footnotetext{
*No proof corrections received from author prior to publication.

${ }^{a)}$ Electronic mail: etcher@cco.caltech.edu
}

calculations ${ }^{5}$ are first performed to establish approximate characteristics of a perfectly periodic $2 \mathrm{D}$ photonic crystal in an optically thin slab.

The properties of the photonic crystal are governed by the 2D geometry, the refractive index of the host dielectric material, the interhole spacing, $a$, and the radius of the air holes, $r$. As mentioned in the introduction we chose to use a triangular array of air holes for our geometry. A $r / a$ ratio of 0.3 was chosen so as to lessen the fabrication tolerances. An effective index of refraction of the slab waveguide layer of 2.65 for the fundamental TE guided mode was calculated using a slab index of 3.4 for InGaAsP at $1.55 \mu \mathrm{m}$. With this effective value of refractive index a $2 \mathrm{D}$ band structure of a triangular array of holes etched into the material is calculated. The band structure revealed the existence of a band gap for TE (electrical field perpendicular to the holes), but no band gap for TM (electric field parallel to axis of holes) polarization as shown in Fig. 1. The center of the band gap is at a normalized frequency of $a / \lambda=0.31$, and the 2D band gap has a width in frequency of $a / \lambda=0.28-0.34$. We will denote the lower frequency band defining the TE bandgap as the "dielectric" band and the upper frequency band as the "air" band. For the light emission peak of InGaAs/InGaAsP quantum wells (QWs) at $1.55 \mu \mathrm{m}$ to be at midgap the hole spacing is $a=500 \mathrm{~nm}$ and the hole radius is $r=150 \mathrm{~nm}$, which is well within the resolution range of electron beam lithography.

With the approximate band structure of the periodic 2D photonic crystal as a guide, we then proceeded to model optical microcavities in which the photonic crystal is used as a mirror. We used a finite-difference time-domain algorithm $^{6-9}$ in 2D and 3D to calculate the quality factor $(Q)$ and the fields of the high $Q$ modes of a variety of optical cavities. In this article we investigate three different geometries for the cavities: A single defect cavity in which one air hole is removed from the 2D lattice, a larger cavity in which a hexagonal area is left unperforated, and a triangular cavity.

A cross-sectional view of the field patterns through the middle of the slab for the three cavity geometries are shown in Fig. 2. From 3D FDTD simulations of the optically thin structure we found that the single defect cavity supports a doubly degenerate dipole mode in which the in-plane radia- 


\section{TE Band Diagram}

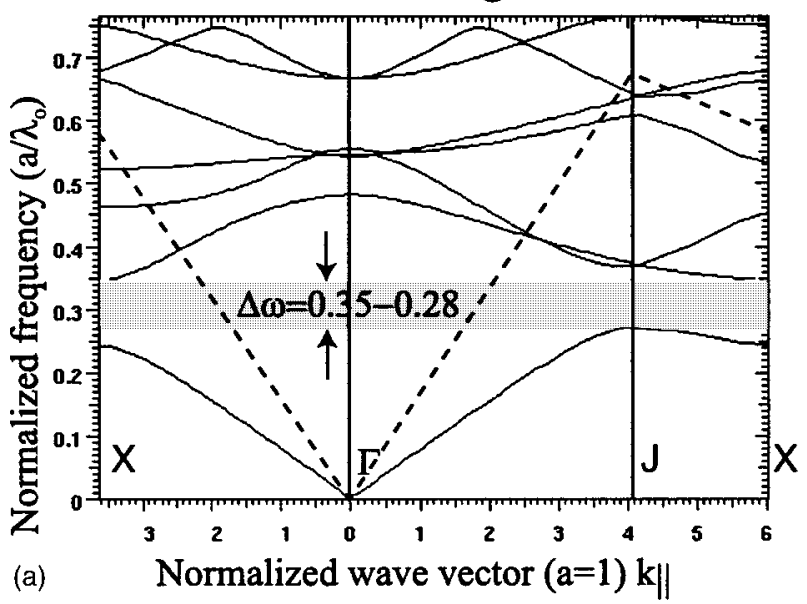

TM Band Diagram

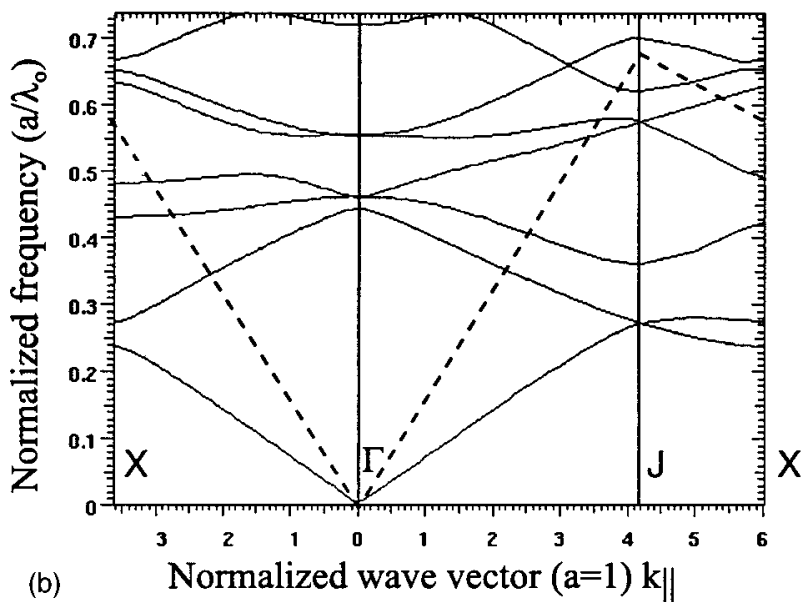

FIG. 1. (a) Band diagram for TE polarized light and (b) corresponding band diagram for TM polarization. The holes have an index of $n_{\text {air }}=1$, the material index is given by an effective index of $n_{\text {slab }}=2.65$, and $r / a=0.3$. The TE band gap extends from a normalized frequency of $0.28-0.35$, and there is no TM band gap. The dashed line represents the light line in air, above which the modes are leaky.

tion is highly directional and well-suited to coupling to an external waveguide as shown in Fig. 2(a). The defect mode has a normalized frequency in the band gap of the photonic crystal and thus is localized to the high dielectric defect region. The $Q$ of the defect cavity is dependent on the number of layers of air holes surrounding the central defect, and on the coupling to the leaky modes which radiate energy normal to the slab. ${ }^{10}$ For the three layers of Fig. 2(a) the $Q$ is approximately 250 . For both the hexagonal and triangular cavities, due to computer memory constraints and calculation time, we performed only 2D FDTD calculations where the slab was assumed infinite in extent in the third direction. The hexagonal cavity supports several high $Q$ modes, one of which, shown in Fig. 2(b), is similar to a whispering gallery type mode. ${ }^{4}$ In Fig. 2(c) we show the magnetic field intensity of a mode of the triangular cavity which is formed from a circulating plane wave which reflects off the middle of each side of the triangle. The $Q$ of the hexagonal cavity and tri-

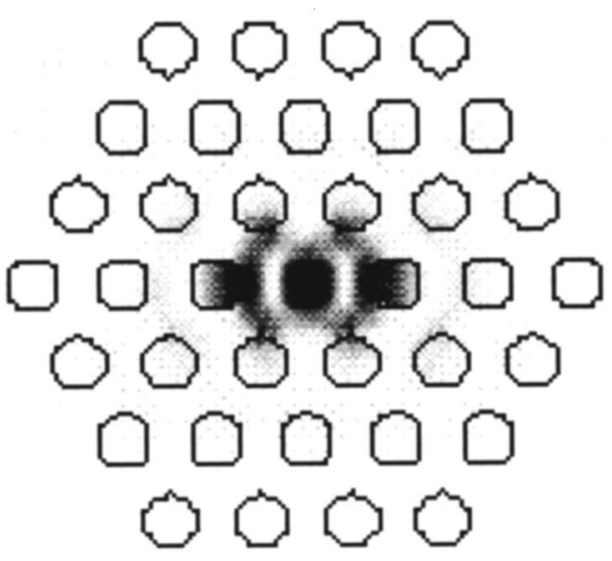

(a)

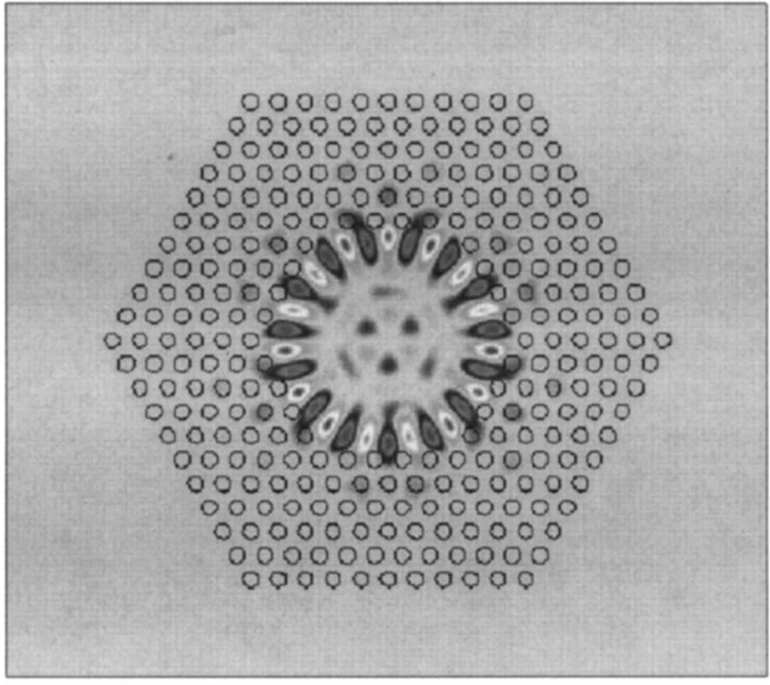

(b)

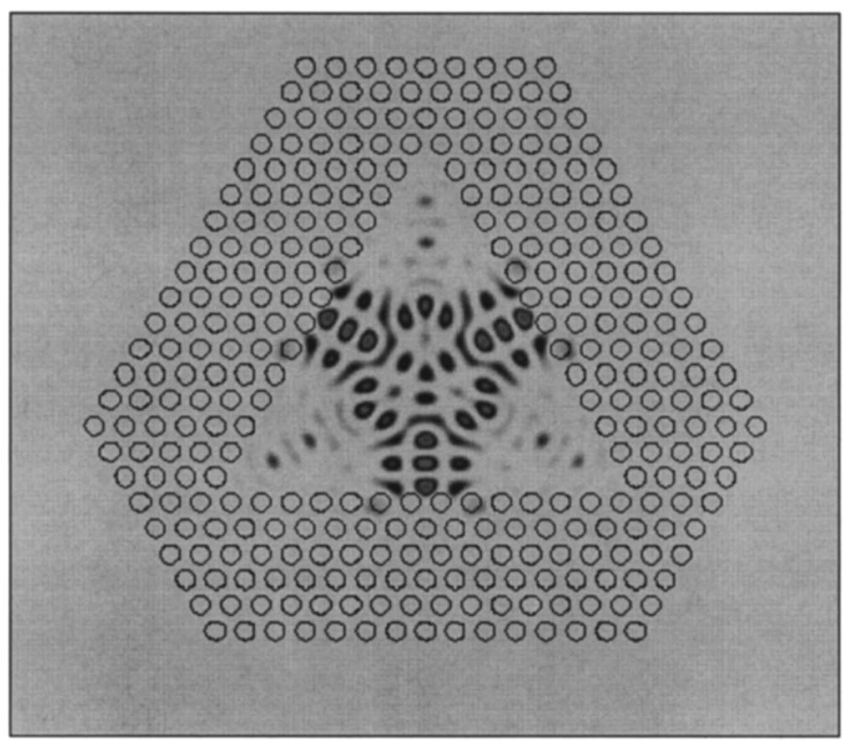

(c)

FIG. 2. (a) 2D slice through the middle of the slab showing the electric field amplitude of one of the defect modes (doubly degenerate). (b) Magnetic field amplitude (scalar field) of a whispering gallery-type mode of the hexagonal cavity. (c) Magnetic field amplitude of a resonant mode of the triangular cavity. 


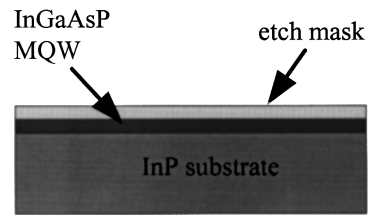

mask patterned with e-beam lithography/IBE/RIE

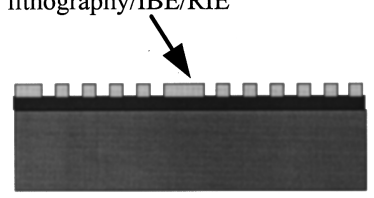

InGaAsP MQW layers etched with CAIBE

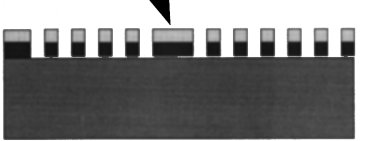

HCL wet etch through the patterned holes

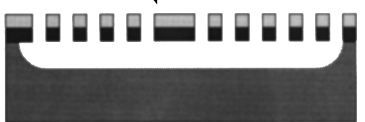

FIG. 3. Schematic process sequence used to define the micromembrane devices. InP is used as a substrate and is selectively attacked by a hydrochloric acid etch to define the final free-standing membrane. The mask layer consists of a silicon dioxide etch mask and PMMA $e$-beam resist.

angular cavity modes shown in Fig. 2 are upwards of 4000, however, since the calculations were done in 2D, scattering losses in the direction normal to the high index slab are neglected.

\section{B. Fabrication procedure}

The fabricated structures incorporated a multi-QD active region inside the high refractive index slab waveguide described in Sec. II A. A structure with six InGaAs QDs separated by InGaAsP barriers, and InGaAsP cap layers on the top and bottom of the multi-QD structure was grown by organometallic vapor phase epitaxy (OMVPE) on an InP substrate. ${ }^{4}$ The epitaxy was designed for peak emission at $1.55 \mu \mathrm{m}$. The total thickness of the multi-QD structure is $180 \mathrm{~nm}$, corresponding to approximately $\lambda / 2$ in the material for $1.55 \mu \mathrm{m}$ emission. A surface mask composed of $60 \mathrm{~nm}$ of $\mathrm{SiO}_{2}$, followed by $5 \mathrm{~nm} \mathrm{Cr}, 50 \mathrm{~nm} \mathrm{Au}$, and $70 \mathrm{~nm}$ of polymethylmethacrylate (PMMA) was deposited on top of this sample. The PMMA resist was then exposed with a Hitachi S-4500 field-emission electron microscope to define the 2D photonic crystals and optical cavities. After developing in cellusolve/methanol, the patterns in the PMMA layer were transferred through the $\mathrm{Cr}$ and Au layers with an $\mathrm{Ar}$ ion beam. This metal mask was used to transfer the lithographic patterns into the $\mathrm{SiO}_{2}$ mask by using a $\mathrm{C}_{2} \mathrm{~F}_{6}$ reactive ion etching process. Finally, the $2 \mathrm{D}$ photonic crystal mirrors were etched through the InGaAs/InGaAsP multiple QW layer by using chemically assisted ion beam etching with an argon ion beam assisted by chlorine reactive gas. After the holes were etched through the InGaAs and InGaAsP layers, the InP substrate was removed by an anisotropic chemical etching with $\mathrm{HCl}$ through the microfabricated holes. This procedure left a suspended membrane of perforated InGaAs/ InGaAsP material and created the membrane microresonators. It is schematically summarized in Fig. 3. Scanning electron micrographs (SEMs) of the three different fabricated cavity geometries are shown in Fig. 4.

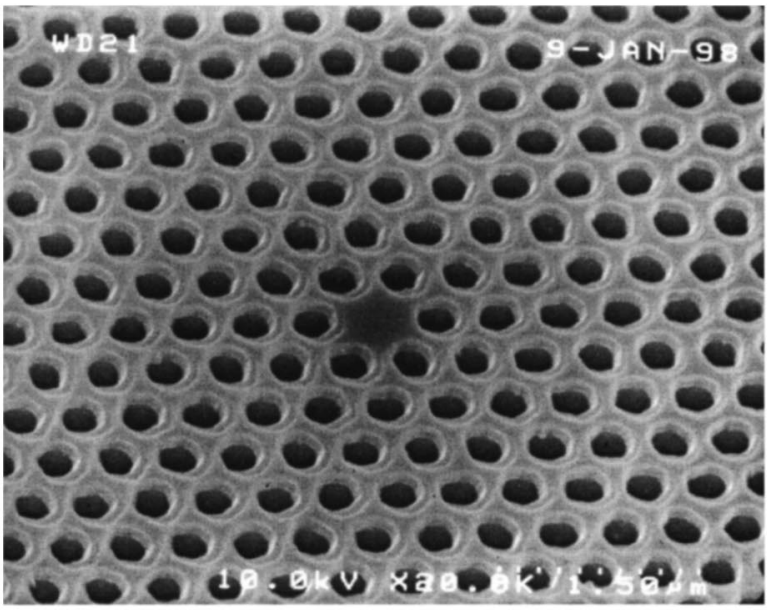

(a)

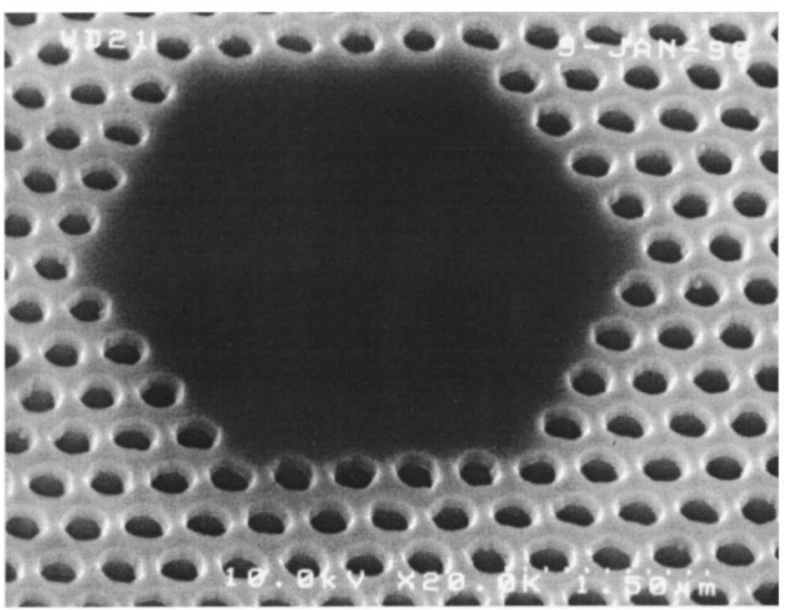

(b)

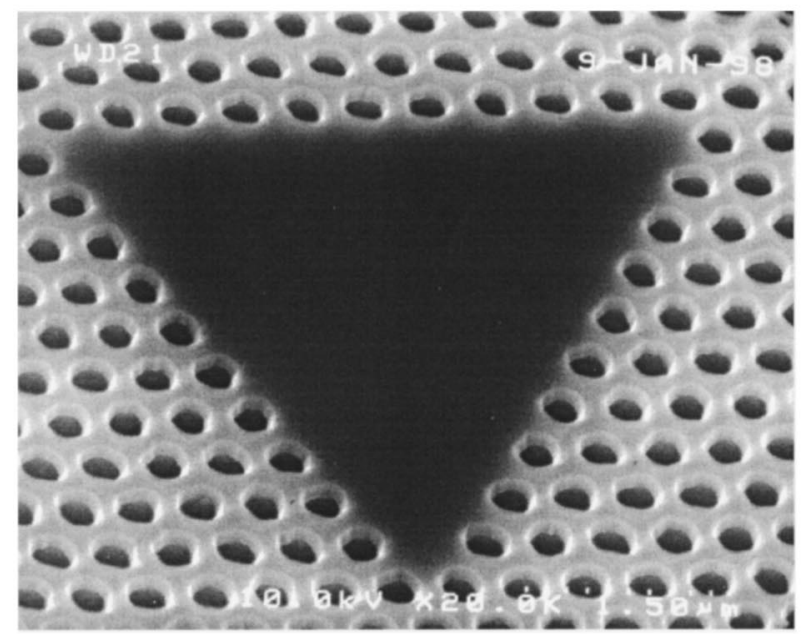

(c)

FIG. 4. SEM micrographs showing examples of microfabricated optical cavities. (a) The defect cavity formed by removing a single hole, and (b) and (c) the hexagonal and triangular optical micromembrane cavities with $\sim 5$ $\mu \mathrm{m}$ lateral dimensions. The InGaAsP membrane contains six InGaAs QWs and is approximately $180 \mathrm{~nm}$ thick.

\section{OPTICAL MEASUREMENTS}

In order to determine the light emission characteristics of these microcavities, we optically pumped them with a 980 


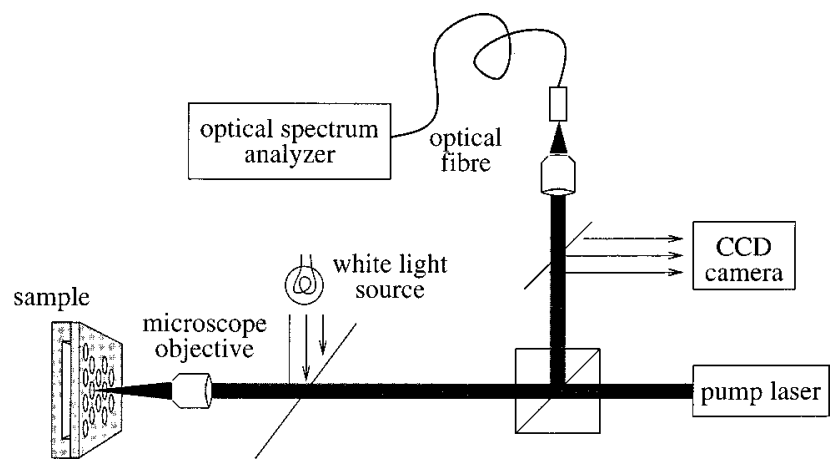

FIG. 5. Spatially resolved optical pumping configuration for probing the photonic crystal microcavities. The pump spot size used was approximately $5 \mu \mathrm{m}$ in diameter.

$\mathrm{nm}$ semiconductor laser focused to a $5 \mu \mathrm{m}$ spot on the sample. The luminescence spectra from the microcavities were measured with an optical spectrum analyzer, which yielded both absolute emission power as well as spectra from 1.2 to $1.6 \mu \mathrm{m}$. A schematic of the measurement set up is shown in Fig. 5. In our structures, the QW emission wavelength was kept constant at $1.55 \mu \mathrm{m}$, set by the initial OMVPE crystal growth step, whereas the spacing and porosity of the photonic crystal were lithographically changed to tune the reflectivity spectra of the photonic crystal. By lithographically tuning the photonic band gap reflectivity spectrum from below the QW emission wavelength to above it, we can evaluate the photonic crystal and infer properties of the optical microcavities formed within them.

In the simplest experiment, we observed the vertical luminescence intensity of a photonic crystal without a cavity as a function of the lattice spacing, i.e., the spacing between holes. This was accomplished by patterning a sample with many $20 \times 20 \mu \mathrm{m}$ areas of photonic crystals with different lattice spacings and hole sizes, and comparing the emission intensity from these. The porosity of each of the photonic crystal lattice constants was measured by SEM analysis and used to normalize the active light emission volume in all structures. Care was taken to factor out any changes in the porosity for fabricated photonic crystals when measuring the total emission intensity. Figure 6 shows the resulting plot of the luminescence intensity as a function of the lattice parameter. From this plot, it is evident that the photonic crystal lattice parameter indeed has a strong influence on the luminescence intensity, and we measured a large increase in the collected light when the TE band gap of the photonic crystal matched the luminescence wavelength. As calculated in Sec. II, a lattice parameter of $500 \mathrm{~nm}$ for a $r / a=0.3$ puts the quantum well emission at $1.55 \mu \mathrm{m}$ approximately at midgap. Above that lattice constant we observed a significant decrease in the luminescence intensity since light can propagate through the patterned material in the guided air band modes. Below the optimum lattice parameter several peaks and valleys are present in the luminescence intensity plot but no sharp decrease in collected emission is observed. As the lattice parameter is reduced further below the optimal value at midgap, the $1.55 \mu \mathrm{m} \mathrm{QW}$ emission is expected to start

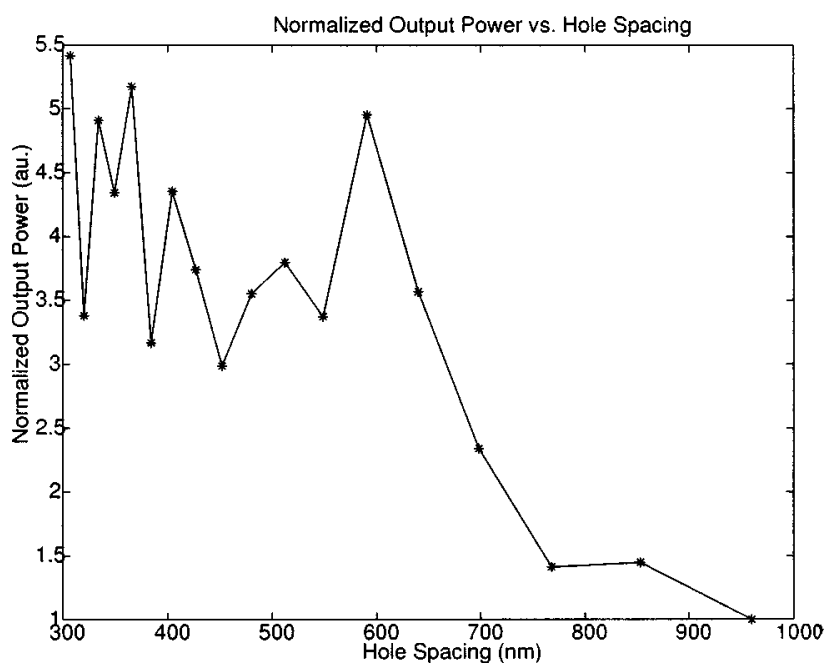

FIG. 6. Plot of the luminescence intensity as a function of lattice parameter from a sequence of photonic crystals which were beam written at different magnifications on the same wafer. The collected light intensity increases by a factor of 4-5 when the photonic crystal is tuned to the emission wavelength of the QWs at $1.55 \mu \mathrm{m}$.

coupling to the guided dielectric band, thus reducing the vertically collected light intensity. More experiments are currently being performed to map out a larger lattice parameter range and to look at the angular emission distribution.

A similar experiment was performed for the hexagonal cavities defined within the photonic crystal. Again, the lattice parameter of the photonic crystal surrounding the cavities was lithographically tuned through the optimum band gap geometry for the $1.55 \mu \mathrm{m}$ emission wavelength, and the luminescence intensity from the membrane microresonator was measured. In the measurement of hexagonal cavities surrounded by photonic crystals with close to optimal geometry, we observed the emergence of resonance peaks in the luminescence spectra as shown in the bottom of Fig. 7. The period of the peaks and valleys in the emission spectra match quite well with the resonance of a cavity with a length matching the diameter of the hexagonal cavity surrounded by two mirrors. In contrast, in the middle spectra of Fig. 7 we show the flat luminescence spectra of a hexagonal cavity with a photonic crystal mirror detuned far from the $1.55 \mu \mathrm{m}$ emission wavelength.

\section{DISCUSSION AND CONCLUSIONS}

It should be noted that although a very porous structure was generated by perforating the multiple QW active area, the vertical emission efficiency from correctly tuned 2D photonic crystals can be significantly higher than that of the unpatterned material. This improvement in the extraction efficiency is a direct result of the more efficient coupling of light out of the plane of the waveguiding membrane. As a result of this microfabricating a photonic band gap tuned to the luminescence wavelength, light is no longer allowed to propagate within the microfabricated membrane. The high luminescence intensity achieved by this geometrically optimum coupling structure was previously predicted, ${ }^{11}$ and is 

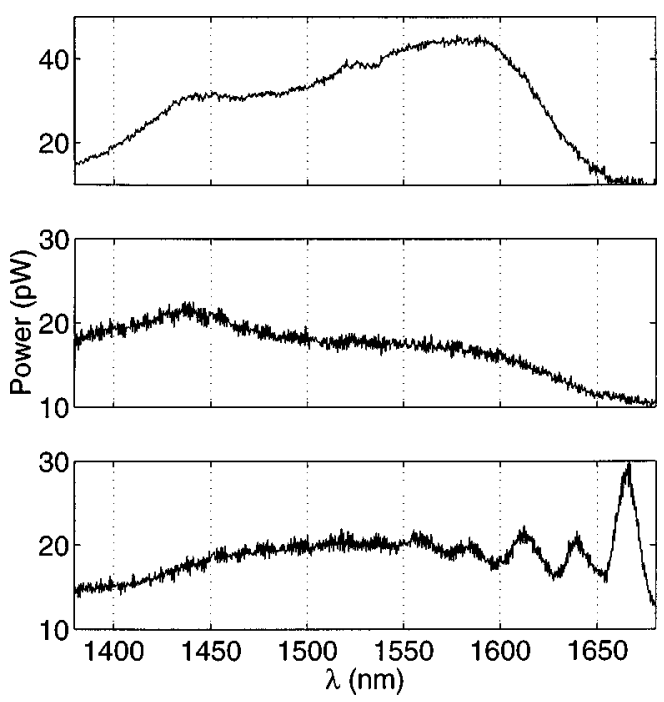

FIG. 7. Spontaneous emission spectra. The top plot shows the control sample emission spectrum from an unprocessed wafer. The middle spectrum shows the emission spectrum from a simple membrane structure with a photonic crystal tuned far out of the emission bandwidth. The bottom spectrum shows resonance peaks in the emission when a correctly tuned photonic crystal is used to form the hexagonal microcavity.

useful in designing highly efficient light emitters. An even more interesting effect expected from very small singledefect microcavities is a change in the spontaneous emission rate. This effect, first predicted by Purcell in $1947,{ }^{12}$ requires the combination of a small optical cavity volume, restricting the number of modes supported by the cavity, and a reasonably high cavity $Q$. The measurement results described here confirm that in the InGaAs/InGaAsP material system the surface damage and sidewall recombination of carriers is low enough to observe such spontaneous emission rate effects. Thus, efficient light generation is possible and the emission characteristics within very small volume optical microcavities can be explored even without surface passivation of the etched sidewalls. Further improvements in the luminescence efficiency are expected from such sidewall passivation and by more effective heat sinking of the membranes.

\section{ACKNOWLEDGMENTS}

The authors acknowledge many helpful technical discussions with T. Fraser-Krauss, M. Borodinski, E. Yablonovitch, and J. O'Brien. Also the authors gratefully thank ORTEL for the epitaxial growth of the wafers used in this experiment. This work was funded by the Army Research Office and the National Science Foundation, whose support is gratefully acknowledged.

${ }^{1}$ J. D. Joannopolous, R. D. Meade, and J. N. Winn, Photonic Crystals (Princeton University Press, Princeton, NJ, 1995).

${ }^{2}$ H. Yokoyama, Science 256, 66 (1992).

${ }^{3}$ T. Baba, IEEE J. Sel. Top. Quantum Electron. 3, 808 (1997).

${ }^{4}$ A. F. J. Levy, S. L. McCall, S. J. Pearton, and R. A. Logan, Electron. Lett. 29, 1666 (1993).

${ }^{5}$ M. Plihal and A. A. Maradudin, Phys. Rev. B 44, 8565 (1991).

${ }^{6}$ M. Bartsch et al., Comput. Phys. Commun. 72, 22 (1992).

${ }^{7}$ D. H. Choi and W. J. R. Hoefer, IEEE Trans. Microwave Theory Tech. 34, 1464 (1986).

${ }^{8} \mathrm{Z}$. Bi, Y. Shen, K. Wu, and J. Litva, IEEE Trans. Microwave Theory Tech. 40, 1611 (1992).

${ }^{9}$ B. D’Urso, O. Painter, J. O'Brien, T. Tombrello, A. Yariv, and A. Scherer, J. Opt. Soc. Am. B 15, 1155 (1998).

${ }^{10}$ P. R. Villeneuve, S. Fan, J. D. Joannopolous, K. Y. Lin, L. A. Kolodziejski, G. S. Petrich, and R. Reif, Appl. Phys. Lett. 67, 167 (1995).

${ }^{11}$ S. Fan, P. R. Villeneuve, J. D. Joannopoulos, and E. F. Schubert, Phys. Rev. Lett. 78, 3294 (1997).

${ }^{12}$ E. M. Purcell, Phys. Rev. 69, 681 (1946). 